\title{
Human vs. AI: Investigating Consumers' Context-Dependent Purchase Intentions for Algorithm-Created Content
}

\author{
Jennifer Rix \\ LMU Munich \\ rix@bwl.lmu.de
}

\author{
Robert Rußell \\ LMU Munich \\ russell@bwl.lmu.de
}

\author{
Alexander Rühr \\ LMU Munich \\ ruehr@bwl.lmu.de
}

\author{
Thomas Hess \\ LMU Munich \\ thess@bwl.lmu.de
}

\begin{abstract}
Increasingly digitalized media consumption is pressuring profitability in the content industry. Technological advancements in the realm of Artificial Intelligence (AI) render the potential to cut costs by applying algorithms to create content. Yet, before implementing algorithm-created content, content providers should be aware of the impact of algorithmic authorship on consumers' intention to purchase said content. Accordingly, this study investigates user attitudes toward algorithmic content creation and their dependence on the underlying utilitarian or hedonic consumption context. In our online experiment $(N=298)$, we find evidence for a positive effect of algorithmic authorship on consumers' purchase intention. Even though the overall purchase intention is context dependent, this algorithm appreciation is independent of the content consumption context. Our study thus suggests that consumers appreciate algorithm-created content. Our results thus provide insights into the benefits of leveraging algorithms in order to maintain content providers' profitability.
\end{abstract}

\section{Introduction}

The content industry faces severe struggles for profitability [1]. Capturing the value of content has, however, become increasingly difficult due to a lower willingness to pay (WTP) for digital content than for an equivalent, but tangible, physical version [2]. At the same time, subscription and advertising earnings are decreasing and can no longer compensate for the reduced WTP for content. Progress in AI-based technologies allows content providers to drive cost efficiency while maintaining content quality [3]. Algorithms for content creation have lately faced increased scrutiny, as they have proven their ability to produce sophisticated, compelling narratives [4]. These algorithms also allow for broadening the scope of offerings by, for example, creating niche and personalized content at low costs [5]. Consequently, algorithms can be leveraged as a powerful tool to help content providers with their struggle for profitability.

However, this anticipated positive effect for content providers only manifests itself if revenues remain stable when algorithms (rather than humans) are implemented as content authors. Extant research suggests a systematic human aversion to algorithms in diverse contexts [6]. Yet, the notion of algorithm aversion is highly debated in the fields of Information Systems (IS) [e.g. 7], Psychology [e.g. 8], and Marketing [e.g. 9]. The studies in these fields provide conflicting evidence. While some find a general aversion toward algorithms, others speak of an appreciation of algorithms [7, 10]. There is already first evidence that, regardless of the content's actual author, the mere disclosure of algorithmic authorship leads to significant differences in consumers' perception of the content [1]. Since consumers' attitudes toward the content drive their intention to purchase it [11], understanding the impact of disclosed algorithmic authorship is pivotal for content providers to leverage algorithms for content creation.

RQ1: Does disclosure of algorithmic authorship affect consumers' intention to purchase content?

Marketing literature contends that intentions to purchase products are dependent on the consumption context $[12,13]$. If the consumption contexts are of a utilitarian nature, consumers follow a superordinate goal [14]. In contexts of a hedonic nature, consumption occurs for its own sake [14]. IS literature also recognizes this distinction between consumption contexts as an important determinant of technology acceptance [15, 16]. In the media industry's content creation, the distinction between the consumption contexts is particularly relevant, because media content can be consumed for both utilitarian and hedonic purposes [11, 17]. Accordingly, algorithms for content creation can be categorized as dual-purpose systems [15]. The relevance of distinguishing between utilitarian and hedonic contexts for consumers' attitude toward algorithms was already suggested in a product recommendation context [18]. Content providers therefore need to understand 
whether consumers' attitude toward algorithmic content creation differs between consumption contexts. If so, this can inform algorithms' application fields.

RQ2: Does the content consumption context affect consumers' appreciation of the author?

We answer our research questions by conducting an online experiment that integrates the effects of i) disclosed authorship and ii) consumption context on consumers' intention to purchase content. We therefore extend the literature on humans' attitudes toward algorithms by presenting a new use case and introducing purchase intention as an outcome measure. Further, we have developed two distinct experimental scenarios that effectively manipulate different consumption contexts for the same dual-purpose system: an algorithm for content creation. We therefore also address a call for inquiry into the relevance of consumption contexts for intentions to purchase content [11].

The remainder of this paper is structured as follows: First, we outline the theoretical foundations of algorithmic content creation, the attitude toward algorithms, and consumption contexts. Subsequently, we derive our hypotheses and explain the experimental approach. We next present and discuss the results, describe the implications, and outline the limitations, as well as future research opportunities.

\section{Theoretical foundations}

\subsection{Algorithmic content creation}

Three technological drivers facilitate the diffusion of algorithmic content creation. The input quality increases constantly due to the improvements in data availability. Together with the advances in Machine Learning as well as Natural Language Generation (NLG), these developments allow algorithms to create compelling content. Algorithms nurtured by these developments can be applied to various content domains. They can be applied to create journalistic articles, produce movies, compose music or even write programming codes $[1,4,19]$.

Algorithmic content creation takes two major forms: template-driven or learning [5]. Template-driven algorithmic content creation refers to filling in designated blanks in pre-written content templates, using rule-based logic [5]. In contrast, learning algorithmic content creation refers to systems taking over the full content production chain, from the data sourcing to constructing elaborate narratives based on data analysis and NLG [20]. The latter systems first start with the collection of data. The data basis could include structured or self-procured recent and historical data from various sources, such as government databases or social media [20]. Second, statistical analyses help identify events and patterns in the data, which are then prioritized on the basis of prior experience and predefined rules. Third, the identified insights' relevance is assessed, while, fourth, NLG is utilized to create the content in line with a predefined style guide [5]. Finally, the content could be published autonomously.

Prior research shows that consumers are unable to distinguish between algorithm-created and humancreated content [3]. In light of the accelerating technological capabilities, the relevance of whether consumers can distinguish between human- and algorithm-created content will diminish even further. Instead, humans' toleration of algorithm-created content will become critical. This toleration is pivotal for marketing algorithm-created content effectively, given that content providers have traditionally sold only human-curated content to consumers.

\subsection{Attitude toward algorithms}

The toleration or appreciation of algorithmic work, which drives the marketability of algorithms' outputs, could be a result of consumers' belief that algorithms are capable of a specific activity. This attitude toward algorithms in a particular task can range from a strong aversion to an appreciation of algorithmic conduct. Previous research has, however, come to contrary conclusions about the circumstances in which algorithm aversion occurs [7, 10].

Algorithm aversion refers to humans' tendency to favor human over algorithmic output, and their reluctance to delegate decisions to algorithmic systems $[8,9]$. In this regard, algorithm aversion suggests that the subjective evaluations of algorithmic systems are systematically distorted. Such distortions may manifest despite the knowledge that a system has complementary capabilities that augment human intelligence [21]. While algorithm aversion traditionally refers to the phenomenon of avoiding reliance on algorithmic systems after witnessing their performance or errors [e.g. 8, 22, 23], other studies have revealed negative attitudes even prior to a confrontation with the system [9, 10, 24]. Extant research suggests that algorithm aversion specifically manifests itself when algorithmic systems assume tasks that are considered innately human [25], are considered subjective [9, 18], and might benefit from human intuition [6]. This preconceived attitude affects users' evaluation of the system, for example, in terms of trust or ability [21].

Contrary to studies that provide evidence of algorithm aversion, other studies find that algorithms are preferred to their human counterparts, therefore suggesting algorithm appreciation $[10,26]$. This notion presumes that aversion is not a default setting, but is instead related to witnessing a malfunction [10]. 
Algorithm appreciation is especially present when the algorithm is known to perform well [24], when tasks suit the algorithm's perceived strengths [9], or its learning ability is displayed during interaction [27]. Algorithm appreciation has even been shown in a journalistic content context, as an AI-based news selection mechanism was appreciated more than a human-curated one [28]. Owing to the mixed evidence regarding algorithm aversion and appreciation, it might be important to consider the fit of a system's perceived capabilities with the underlying task $[9,29]$.

Consumers may, nevertheless, also be indifferent toward algorithms in certain domains, suggesting neither an algorithm aversion nor appreciation. Extant research on attitudes toward algorithms is often set in medical or actuarial domains [2, 27]. Given the taskdependency of algorithm aversion, different application domains could also account for distinct attitudes [9]. In the content-creation domain, attributing authorship to an algorithm has been shown to affect the content's evaluation, even if only the author byline was manipulated rather than the actual article [1]. The latter suggests the prevalence of cognitive preconceptions toward algorithm-created content. Algorithmic authorship has a positive impact on consumers' perceptions of content's objectivity, but algorithmcreated content simultaneously induces less emotional involvement and is attributed with exhibiting less expertise [30, 31]. Further, there are contrasting studies concerning the impacts of algorithmic authorship on the perceived credibility of content $[32,33]$. Although the direction of effects remains unclear due to the mixed evidence, these studies indicate that consumers take an interest in whether an algorithm was involved in the content creation process.

\subsection{Utilitarian vs. hedonic contexts}

The relevance of the consumption context in content consumption can be derived from the uses and gratification theory, a motivational paradigm rooted in communication science. This theory is used to explain (online) media consumption and identifies information and entertainment as the primary dual motivators for the consumption of content [17, 34]. Further, recent research addresses the prevailing neglect of the consumption context when the intention to purchase content is analyzed and calls for research on this matter [11]. These insights align well with consumption contexts' conceptualization in Marketing and IS literature $[14,15]$.

Marketing literature distinguishes between utilitarian and hedonic products. Consumers are said to have utilitarian consumption purposes when their consumption is associated with a superordinate goal, but hedonic consumption purposes when their consumption is associated with enjoyment [14]. Purchasing products for utilitarian purposes is justified more easily for consumers while purchasing products for hedonic purposes is often associated with guilt [35]. Consumers' comparably higher WTP, speeds of purchase, and purchase intentions in utilitarian contexts also reflect this $[12,13,36]$.

The underlying consumption contexts are also established in the context of technology acceptance in IS research [15, 16]. While utilitarian systems are closely associated with usefulness, hedonic systems are linked to enjoyment [15]. Systems' utilitarian vs. hedonic nature is decisive when investigating their acceptance [16]. Some systems can further be used in utilitarian as well as hedonic contexts, so-called dualpurpose systems [15].

When merging the insights on consumption motives from Marketing and technology acceptance literature with the prevalence of information and entertainment as the dual motivators of content consumption, it is possible to establish that content can be used for both, utilitarian and hedonic consumption. Thus, while individuals may, on the one hand, consume content to fulfill a task associated with a specific goal, such as gaining knowledge of a topic, they may, on the other hand, merely consume content to pass time.

Since the consumption context divides the overall content consumption domain, researchers should also take it into account when investigating consumers' attitudes toward algorithm-created content. Similar to dual-purpose systems, algorithms can create content with utilitarian, information-focused purposes, as well as hedonic, entertainment-focused purposes [1, 15]. Prevailing literature on the attitude toward algorithms reinforces the need for this distinction. Humans' preference for utilizing AI recommenders has been shown to be higher if there is an underlying utilitarian consumption goal [18]. Further, for highly subjective tasks, consumers perceive algorithms as lacking the required capabilities, while in objective, quantifiable tasks, algorithms are perceived as suitable [9]. Hereby, objective tasks can be rather associated with utilitarian consumption contexts and vice versa.

\section{Hypotheses development}

The acceptance of technology is fostered by the fit of its perceived capabilities to its specific purpose [29]. It is therefore important to consider consumers' perceptions of the capabilities of algorithmic content creation systems and to match these with the capabilities required for content creation. Algorithm aversion is specifically present when a machine assumes tasks thought to require intuition or when used for a subjective 
task [6, 9]. In contrast, algorithm appreciation rather manifests in objective tasks in which the algorithm was perceived to perform well $[9,10]$.

With creating content, algorithms assume a task associated with human intuition and in which ethical conduct is highly relevant [25]. Further, human authors are distinguished in respect of two major capabilities. First, they distinguish themselves from algorithms by using creativity, as well as uncommon and colorful words, to make their texts vivid [5]. Second, an original writing style that provides more than what is rationally required, makes an author stand out [37]. In contrast, algorithms are attributed a lack of originality, authenticity, and creativity due to their lack of conscience [22, 38]. Furthermore, positive effects of disclosed algorithmic authorship on the perception of content, and hence indications for perceptions of suitable capabilities of algorithms, have only been shown in data-driven domains [1]. These positive effects may no longer prevail in broader domains. Therefore, despite the mixed evidence, algorithm aversion is hypothesized to prevail in the content creation domain.

This aversion may eventually manifest itself in user behavior. Extant research on attitudes toward algorithms generally investigates system use, adherence to recommendations, or evaluation of the system as outcomes [21]. This 'system use' context is not directly transferable to consumers' acceptance of algorithmcreated products. Rather, given that firms' ultimate goal is to monetize their products, the intention to purchase these products is a relevant outcome measure. For example, a study has shown that disclosing a chatbot's algorithmic identity has a negative effect on its effectiveness as an algorithmic sales agent [39]. Therefore, consumers' willingness to purchase content can be considered to reflect consumers' attitudes toward algorithms in the respective domain. We therefore hypothesize that algorithmic authorship impacts consumers' intention to purchase content negatively.

H1: Algorithmic authorship (compared to human authorship) affects the intention to purchase content negatively.

The specific content consumption context, or the consumption purpose, is of particular relevance when investigating consumer attitudes [18]. In common product settings, studies have shown that the intention to purchase utilitarian products is higher than purchasing hedonic products $[12,13]$. In a similar vein, studies have shown that content-providing hedonic apps are purchased at a slower rate than their utilitarian counterparts, given a free version of this app [36]. This utilitarian vs. hedonic distinction in purchase intentions is likely to extend to the content consumption domain. We therefore hypothesize that a utilitarian consumption purpose has a positive effect on the content purchase intention.

H2: A utilitarian consumption context (compared to a hedonic consumption context) affects the intention to purchase content positively.

In general, people are more likely to rely on an algorithm's recommendations if they have a utilitarian consumption goal [18]. The prevalence of algorithm aversion or appreciation is therefore assumed to be distinct according to the respective consumption context of dual-purpose systems.

In the content domain, one cannot directly deduce consumption contexts from an article's topic. Nevertheless, the variance in the perceptions of contents produced by algorithms with regard to the underlying topic gives a first indication that context may matter in respect of attitudes toward content-creation algorithms [40]. It can therefore be assumed that consumers' underlying consumption context is of relevance for their attitude toward using algorithms for content creation.

The algorithm's capabilities' perceived fit with the task of creating content for the respective consumption context may drive consumers' attitude toward algorithmic content creation. Utilitarian consumption contexts require plain, easily accessible content in order to facilitate the extraction of information [40]. Research has further shown that consumers regard the objectivity and credibility of algorithmic content creation systems as superior to those of human journalists [30, 32]. Similarly, algorithms are also generally perceived as more objective than humans [9]. Algorithm-created content may therefore be perceived as more suitable in situations in which objective content is essential. This is especially prevalent in utilitarian consumption. In contrast, algorithmic content creation may be perceived as less suitable for hedonic consumption. Algorithms are ascribed a lack of creativity and serendipity, which are both important drivers of consumers' perceived enjoyment of content $[37,38]$. Accordingly, in line with the increase in algorithm aversion in subjective contexts [9], we hypothesize that a utilitarian consumption context weakens algorithmic authorship's hypothesized negative effect on consumers' intention to purchase content.

H3: A utilitarian consumption context moderates the effect of algorithmic authorship on the intention to purchase content positively.

\section{Methodology}

\subsection{Experimental design and procedure}

To test the hypothesized relationships between authorship, consumption context, and purchase intention, we conducted an online experiment with a 2 
(authorship: algorithmic vs. human) x 2 (consumption context: utilitarian vs. hedonic) between-subjects design in a content consumption setting. Figure 1 summarizes the full experimental procedure.

After the instructions of step 1, we assigned the participants randomly to either the utilitarian or hedonic consumption context treatment in step 2. The overall idea of framing different consumption scenarios to manipulate consumption contexts is in line with Botti and McGill [14], who analyzed the impact of consumption purposes on consumer satisfaction.

Per definition, a manipulation in respect of utilitarian consumption requires providing a consumer with a higher instrumental goal associated with performing an activity [14]. We created this scenario by introducing the utilitarian treatment group participants to a scenario in which they are a university student assigned to write a graded paper on the touristic developments in remote regions of the world. We also mentioned that this assignment would have a significant impact on their job prospects. In addition, we informed the participants that we would provide them with three articles for their research and that they should study these for at least five minutes to be able to answer a follow-up content question after each article. These attention checks based on stimulus material are valid tools for ensuring that participants engage with the content to reinforce the scenario's 'research' task.

In contrast, a hedonic consumption purpose is characterized as non-instrumental [14], which means an activity is conducted for its own sake and the enjoyment related to this activity. Consequently, the manipulation needed to ensure that no specific goal motivated the participants to consume the content. We therefore informed them that they would face a waiting time of five minutes. We also notified them that they would be given the opportunity to voluntarily read three articles to pass this waiting time. Those participants not wishing to consume content during the wait could also proceed with the experiment after waiting five minutes. Hereby, the actual reading of articles by participants in the hedonic treatment groups was not relevant for this study's outcome. In order to achieve procedural symmetry regarding the attention checks without implying a consumption goal, we informed the participants that they would be given the opportunity to indicate how much or little they liked the articles after reading each one. To prevent the hedonic scenario from gaining a utilitarian nature due to knowledge-based questions, the attention checks only reflected personal opinion and were voluntary. On the whole, this setup ensured that the articles would only be consumed for the sole purpose of reading them for enjoyment.

In step 3, we familiarized the participants with the author of the articles they were about to read. We not only provided short descriptions of the respective author but also illustrated the steps involved in the content creation process. We introduced the human author treatment groups to a standardized journalistic process and a press code of conduct, while we informed the algorithmic author groups about the processual conduct and technologies that enable algorithmic content creation. The manipulations as of steps 2 and 3 were randomized, resulting in four treatment groups.

In step 4, all the participants were given the same content stimulus material, which comprised three articles on travel destinations. We intentionally used the same articles as doing so improves external validity and allows for measuring the algorithmic authorship's impact rather than its technological capabilities. The leisure-associated topic of these articles, travel, was used to distinguish this study from prior research since most studies on algorithmic authorship only include highly data-driven articles [1]. This allowed this study to extend the range of application domains of contentcreation algorithms. An embedded timer ensured that participants remained in step 4 for at least five minutes. Afterward, participants proceeded to step 5, the postexperimental questionnaire.

\subsection{Measures}

We carefully adapted established scales to our research context to ensure content validity. We measured participants' willingness to purchase content on the basis of the purchase intention construct by Pennington et al. [41]. To test whether our treatment led to the intended manipulations, i.e. whether the effects on

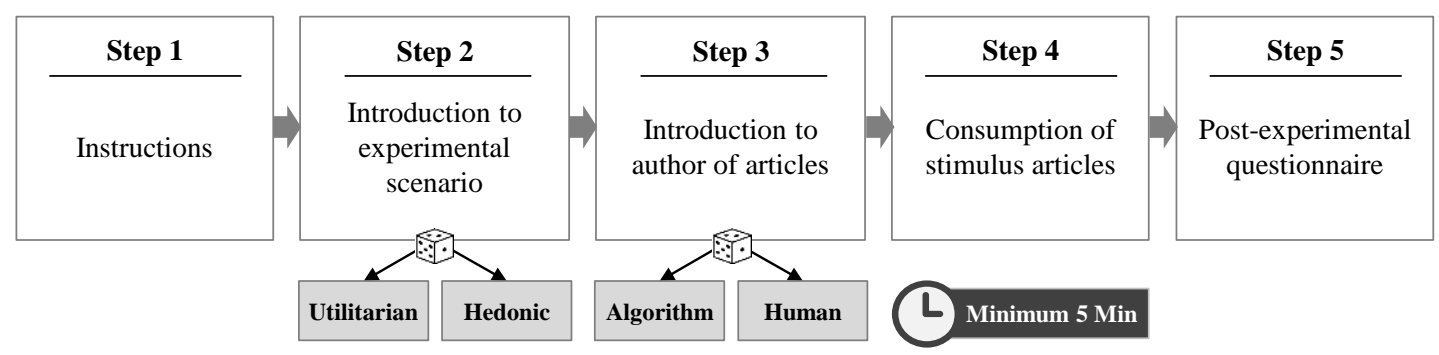

Figure 1. Experimental procedure 
purchase intention could be attributed to our treatments, we used a consumption context construct adapted from $\mathrm{Xu}$ et al. [42], as well as a metric to assess the perceived automation adapted from Holtgraves et al. [43] and Schuetzler et al. [44]. To control for potential systematic differences in our treatment groups, we further captured personal innovativeness [45] and demographics such as age, gender, profession, and income.

\subsection{Data collection and sample}

Before commencing with the final data collection, we conducted nine pre-tests. We recruited the study participants from a mailing list of students from a large, public European university, as well as via a peer-to-peer survey platform. Since samples primarily composed of students are frequently used in related studies [e.g. 46], they are also suitable for this study. We incentivized participation by means of a lottery for three shopping vouchers worth $€ 50$ each. Overall, a total of 503 subjects started the experiment, 300 of whom completed it. We analyzed the data for unengaged responses as indicated by the standard deviation of the participants' Likertscale responses. Two respondents were excluded due to a standard deviation of zero across all the measurement constructs. The sample for analysis therefore included 298 completed questionnaires with the following group sizes: algorithmic/utilitarian, $\mathrm{n}=70$; algorithmic/ hedonic, $\mathrm{n}=78$; human/utilitarian, $\mathrm{n}=72$; and human/ hedonic, $\mathrm{n}=78$. The sample comprised $62.42 \%$ females, with an average age of $25.54(\mathrm{SD}=7.79)$. Their age ranged from 17 to 69 years. The sample mostly included students $(80.87 \%) .78 .73 \%$ of the participants who disclosed their income earned less than $€ 1,500$ a month.

\section{Results}

\subsection{Validation of experimental setting}

To ensure our randomization's effectiveness, we tested for significant differences between the four treatment groups regarding gender, profession, and income, finding no significant differences (all $\mathrm{p}>0.1$ ). Analyses of variance showed that there were no significant differences between the treatment groups regarding age $(\mathrm{F}=0.89, \mathrm{p}>0.1)$ and personal innovativeness $(\mathrm{F}=0.56, \mathrm{p}>0.1)$. We therefore assume that participants' demographics and characteristics were balanced across the treatment groups.

The manipulation checks supported our treatments' intended effects. The utilitarian treatment groups' participants perceived the underlying experimental scenario significantly more utilitarian than the hedonic treatment groups did $(\mathrm{F}=67.75, \mathrm{p}<0.001)$. In contrast, the hedonic treatment groups' participants perceived the scenario significantly more hedonic than the utilitarian treatment groups did $(F=43.84, p<0.001)$. In terms of the authorship's effective manipulation, we found significant differences $(\mathrm{F}=142.88, \mathrm{p}<0.001)$ between the perceived automation across the human $(\mathrm{M}=3.20$, $\mathrm{SD}=1.26)$ and algorithmic authorship $(\mathrm{M}=4.98, \mathrm{SD}=$ 1.31) groups.

We included authorship (algorithm vs. human) as well as consumption context (utilitarian vs. hedonic) as binary variables in our statistical model. We conducted a confirmatory factor analysis of the latent variable purchase intention to ensure its validity. We then calculated Cronbach's alpha (CA) and the composite reliability (CR) to ensure internal consistency reliability. The latent variable surpassed the threshold of 0.7 for CA and CR. The factors loadings also exceeded the threshold of 0.7 , implying indicator reliability [47]. Lastly, convergent validity was ensured as the average variance extracted (AVE) value exceeded 0.5.

\subsection{Hypotheses testing}

We assessed the statistical significance of the effects by means of two-sample t-tests, which provide a good fit due to the categorical independent variables. Hereby, no posthoc tests were required due to the binary nature of the independent variables [48].

First, a significant main effect of the disclosed author on the latent variable of purchase intention was revealed; $\mathrm{t}(296)=-2.11, \mathrm{p}=0.036$. It can therefore be inferred that consumers have a higher purchase intention for algorithm-created than for human-created content. In contrast to the hypothesized presence of algorithm aversion, algorithm appreciation seems to be applicable in this context. Accordingly, we found no support for $\mathrm{H} 1$, as the effect exhibits a different direction than the hypothesized one. Second, the consumption purpose had a significant main effect on the purchase intention; $\mathrm{t}(296)=-6.33, \mathrm{p}<0.001$. Consequently, we can infer that consumers have a higher purchase intention in a utilitarian consumption context. Accordingly, H2 was supported.

Figure 2 depicts the means of the sum of purchase intention items detached from purchase intention as a latent variable. The comparison of the purchase intention means for algorithmic $(\mathrm{M}=2.37, \mathrm{SD}=1.45)$ and human $(\mathrm{M}=2.05, \mathrm{SD}=1.16)$ authorship, as well as utilitarian $(\mathrm{M}=2.68, \mathrm{SD}=1.43)$ and hedonic $(\mathrm{M}=1.78$, $\mathrm{SD}=1.05)$ consumption contexts, further illustrates the direction of the effects.

Third, a regression was used to test for an interaction effect. We found a non-significant interaction between the disclosed author and consumption context; $\mathrm{t}(296)=0.19, \mathrm{p}>0.1$. 
Consequently, algorithmic authorship's effect on purchase intention is not moderated by the respective consumption context. H3 is therefore not supported. Figure 3 summarizes the effects that the mean comparisons $(\mathrm{H} 1, \mathrm{H} 2)$ and the regression for the interaction effect $(\mathrm{H} 3)$ reveal.
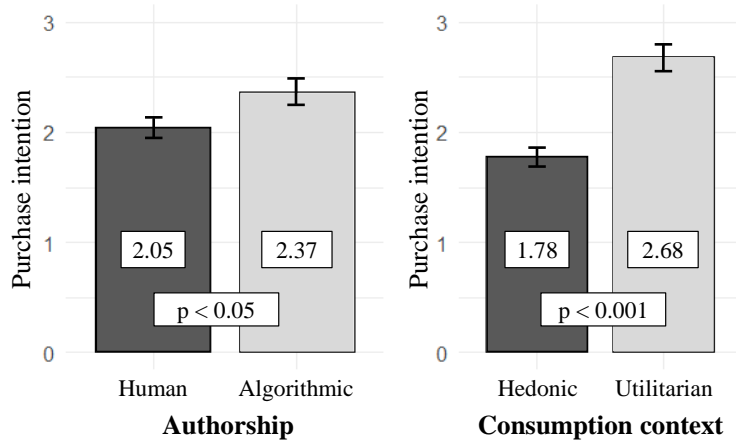

Figure 2. Mean comparisons

\section{Discussion}

In the content creation domain, we find that the disclosure of algorithmic authorship has a positive effect on consumers' intention to purchase content. This indicates that instead of the presumed algorithm aversion, algorithm appreciation applies. Overall, the algorithm appreciation in this domain may be explained by consumers i) perceiving algorithms' capabilities to fit well to the challenges of producing content, ii) witnessing the algorithm perform well, or iii) being transparently informed about the functionality of algorithmic content creation systems.

First, regarding algorithms' perceived capabilities, prior research has shown that algorithms evoke aversion if they are perceived as incapable of performing well in an innately human, and hence subjective, task [9]. In the context of this study, the task of creating content for travel destination articles could be perceived as more objective than initially assumed. Consumers might also appreciate the perceived improvements in objectivity and the reduced bias in the content that the algorithmic authorship induces [30, 31]. Algorithms' presumed capabilities could therefore indeed be perceived as suitable for the task [9]. Thus, in consumers' overall evaluation of the content, the objectivity and bias considerations could outweigh the perceived negative associations with algorithmic authorship, such as the lack of expertise or emotional involvement [30, 33]. This is also in line with another study in a journalistic domain, which revealed algorithm appreciation for news recommender systems [28].

Second, the participants have observed an algorithm performing error-free and exhibiting elaborate skills when creating content. Studies have shown that seeing algorithms perform well evokes algorithm appreciation [e.g. 26] and is, in general, a driver of system acceptance. In support, the contentcreation algorithm as of this study may have outperformed participants' expectations. According to the expectation disconfirmation theory, if a technology outperforms a person's expectations, for example in this context in terms of making no errors and using proper syntax, the person's post-adoption satisfaction is improved [49]. Owing to their unfamiliarity with algorithm-created content, consumers may have lower expectations toward algorithm-created content. The absence of errors in the algorithm-created content could therefore have led to a higher satisfaction and, eventually, also intention to purchase [24]. Nevertheless, algorithmic authorship's positive effect may deteriorate over time as consumers get used to it. Similarly, its positive effect could disappear, or even be reversed, when consumers witness the algorithm err, even if the error is as simple as a spelling error [8].

Third, studies have shown that explanations of an algorithm's functionality reduce aversion [23]. Providing information about a system's inner workings improves process transparency [50, 51]. Logg, Minson and Moore [10] explicitly highlight the importance of process transparency for algorithm appreciation. The underlying study explains the algorithmic content creation process in detail. Process transparency may therefore be regarded as a potential driver of the uncovered algorithm appreciation. Further, the role of

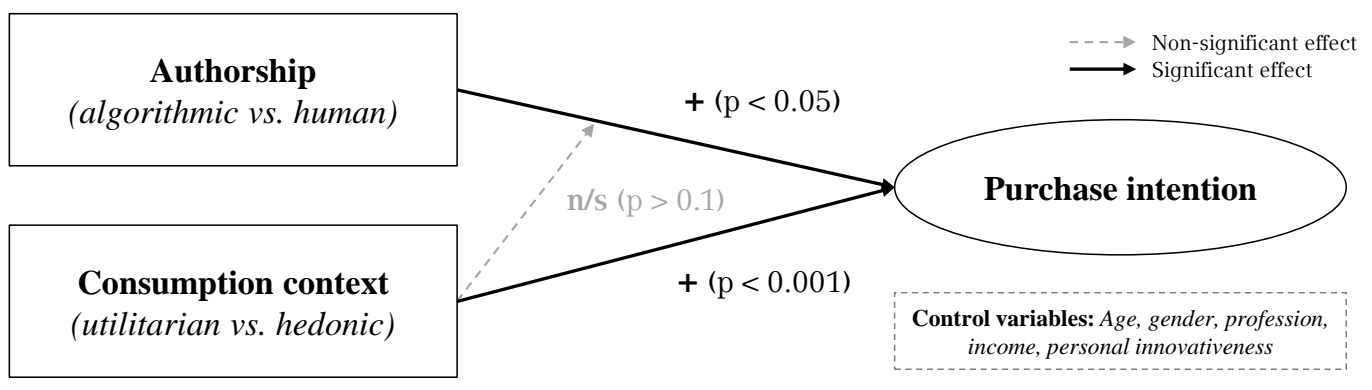

Figure 3. Research model and significance of effects $(\mathrm{N}=298)$ 
information transparency, which allows consumers to understand which input data the algorithm used for its product, should also be taken into account [52]. Yet, this study did not account for this type of transparency.

Evidence for the positive effect of a utilitarian context has added a new dimension to inquiries into the purchase intentions for content, which is a pivotal notion for marketing content effectively [11]. The insights into consumption context may also be transferable to content topics. The topic can indeed suggest a more utilitarian or hedonic consumption experience (e.g. finance vs. lifestyle content). Nevertheless, the topic and the consumption context cannot be used interchangeably. Articles on any topic could be consumed in utilitarian as well as hedonic contexts. The likelihood of a particular topic being consumed for a utilitarian or hedonic purpose varies with consumers' personal preferences.

The consumption context has not been shown to moderate disclosed authorship's effect on consumers' purchase intention. Most of the existing research on algorithm-created content has used data-driven articles, which are often associated with utilitarian consumption contexts [1]. Our study deliberately picked travelfocused stimulus material. Travel is mostly associated with enjoyment and could be considered a soft, nondata-driven topic. Consequently, our study allows for the inference that algorithmic content creation systems are also accepted - and could even be monetized - in less data-driven areas of application. Further, the lack of a moderating effect of consumption context on consumers' technology acceptance revealed in this study contrasts extant research that shows that a utilitarian goal leads to a higher likelihood of choosing an AI recommender [18]. Consumers' indifference to contexts might be rooted in the distinct type of algorithm investigated, as the underlying study examined a performative instead of an advisory algorithm. Consequently, the type of algorithm can be assumed to be relevant when assessing attitudes toward algorithms.

\section{Theoretical and practical implications}

First, we uncover algorithm appreciation and, therefore, consumers' acceptance of algorithms in the content creation domain. Herewith, we contribute to Marketing and IS literature on consumers' attitude toward algorithms [7, 9]. We introduce the measurement of attitude toward algorithms by means of purchase intention to investigate the marketability of algorithmcreated products [21].

Second, we introduce a novel use case to the application domains of research on attitudes toward algorithms [21]. Having another study show algorithm appreciation for an advisory algorithm in the content domain allows the inference that a system's application domain might steer attitudes toward algorithms [28]. Subsequent studies should therefore carefully take the research setting's potential effects into account. Moreover, our study is, to the best of our knowledge, the first to find algorithm appreciation when simulating a performative algorithm, i.e. an algorithm that exhibits a high degree of autonomy in task execution [21].

Third, also the distinction of consumption contexts has caught attention in Marketing and IS [15, 16, 18] as well as content-focused literature [11]. The significant effect of consumption context on purchase intention suggests that the consideration of context is essential when inquiring into the content domain. A utilitarian consumption context's positive effect on consumers' purchase intention in the content domain could also be generally applicable. Utilitarian framing might therefore improve the purchase intentions for dual-purpose systems. Further, this study introduces a novel and effective methodological approach to manipulate consumption contexts for dual-purpose systems [15]. Despite the challenge of creating purely hedonic contexts in an online experiment, the participants' need to pass time was effectively operationalized to create a hedonic consumption context. Thus, we successfully transferred methodological insights from Marketing to IS research [14].

Last, owing to the absence of a moderating effect or the presence of a weak one, we suggest that the content consumption context is less relevant for attitudes toward algorithms in content creation. Our study therefore adds to extant literature by indicating that the attitude toward algorithms is either detached from or only weakly influenced by the consumption context of algorithmcreated products. Content creation algorithms' dualpurpose nature is hereby further reinforced [15].

The findings of this study are also highly practically relevant for content providers who monetize content as part of their core business. While this study focuses on textual content, the findings also have implications for other types of content that algorithms can create, such as video and audio content or even codes [4, 19].

First, as there was an indication of a higher intention to purchase algorithm- rather than humancreated content, leveraging algorithms for content creation is an attractive option. Algorithmic authorship is unlikely to affect revenues negatively or can even be operationalized as a sales argument for the content. Further, algorithmic authorship can and should be communicated transparently. Nevertheless, when implementing algorithms for content creation, errors should be regarded cautiously. To prevent a reversal from appreciation to aversion due to witnessing errors [8], the algorithm's performance should be closely monitored. In addition, the respective content creation algorithm's functionality should be disclosed, as such 
explanations can foster appreciation [23]. Content providers face no direct costs for establishing process transparency [50]. They could also consider publishing the data used, hence establish information transparency [52]. Despite these upsides, content providers should keep liability and bias considerations in mind [25].

Second, consumers were shown to be more willing to purchase content if they consume content for utilitarian purposes. This highlights the need for content providers to identify consumers' underlying consumption context by, for example, uncovering patterns in reading habits. Based hereon, content providers could implement a freemium paywall that adjusts its configuration to the underlying consumption context. In this notion, based on the subordinate relevance of the consumption context for consumers' attitudes toward content-creation algorithms, content providers should further consider applying algorithms in non-data-driven, soft domains.

\section{Limitations and future research}

Despite its careful design, this study has certain limitations, which yield potential for future research. First, manipulating the consumption context bears challenges, as participants are artificially introduced to a stylized environment. Although we showed the manipulation's effectiveness, the consumption context's effect could be even more pronounced in reality, especially in a hedonic context. Future research could address the consumption context in a more controlled environment, such as in a laboratory. Hereby, it could be ensured that the waiting time cannot be used for activities other than reading or waiting. Second, participants were asked whether they would purchase free access to all of the author's articles for a small fee. Given the algorithmic production, participants might have set a lower price anchor in the case of algorithmcreated content. To control for this eventuality, future research should investigate WTP in conjunction with the purchase intention to provide a quantitative price anchor. Third, this study only provides information about disclosed authorship's effect on the purchase intention, but does not attempt to find the drivers or mediators of this effect. Future research should investigate the perceptions of the content as well as the impact of process and information transparency. In this notion, investigating the effects of joint human and algorithm authorship, which is also frequently observable in practice, might yield interesting insights [4]. Lastly, we made use of a student sample, while the broad population might be more technology averse. A broader sample could improve the external validity further.

\section{References}

[1] A. Graefe, and N. Bohlken, "Automated journalism: A meta-analysis of readers' perceptions of human-written in comparison to automated news", Media and Communication, 8(3), 2020, pp. 50-59.

[2] B. Berger, C. Matt, D.M. Steininger, and T. Hess, "It is not just about competition with "free": Differences between content formats in consumer preferences and willingness to pay", Journal of Management Information Systems, 32(3), 2015, pp. 105-128.

[3] C. Clerwall, "Enter the robot journalist", Journalism Practice, 8(5), 2014, pp. 519-531.

[4] The Guardian, "A robot wrote this entire article. Are you scared yet, human?", https://www.theguardian.com/ commentisfree/2020/sep/08/robot-wrote-this-article-gpt-3,

accessed 07.02.2021.

[5] C. Zydorek, Grundlagen der Medienwirtschaft: Algorithmen und Medienmanagement, Springer, 2018.

[6] S. Highhouse, "Stubborn reliance on intuition and subjectivity in employee selection", Industrial and Organizational Psychology, 1(3), 2008, pp. 333-342.

[7] B. Berger, M. Adam, A. Rühr, and A. Benlian, "Watch me improve - Algorithm aversion and demonstrating the ability to learn", Business \& Information Systems Engineering, 63(1), 2021, pp. 55-68.

[8] B.J. Dietvorst, J.P. Simmons, and C. Massey, "Algorithm aversion: People erroneously avoid algorithms after seeing them err", Journal of Experimental Psychology: General, 144(1), 2015, pp. 114-126.

[9] N. Castelo, M.W. Bos, and D.R. Lehmann, "Taskdependent algorithm aversion", Journal of Marketing Research, 56(5), 2019, pp. 809-825.

[10] J.M. Logg, J.A. Minson, and D.A. Moore, "Algorithm appreciation: People prefer algorithmic to human judgment", Organizational Behavior and Human Decision Processes, 151, 2019, pp. 90-103.

[11] D. O’Brien, C.-M. Wellbrock, and N. Kleer, "Content for free? Drivers of past payment, paying intent and willingness to pay for digital journalism", Digital Journalism, 8(5), 2020, pp. 643-672.

[12] E.M. Okada, "Justification effects on consumer choice of hedonic and utilitarian goods", Journal of Marketing Research, 42(1), 2005, pp. 43-53.

[13] U. Khan, and R. Dhar, "Price-framing effects on the purchase of hedonic and utilitarian bundles", Journal of Marketing Research, 47(6), 2010, pp. 1090-1099.

[14] S. Botti, and A.L. McGill, "The locus of choice: Personal causality and satisfaction with hedonic and utilitarian decisions", Journal of Consumer Research, 37(6), 2011, pp. 1065-1078.

[15] J. Wu, and X. Lu, "Effects of extrinsic and intrinsic motivators on using utilitarian, hedonic, and dual-purposed information systems: A meta-analysis", Journal of the Association for Information Systems, 14, 2013, pp. 153-191.

[16] H. Van der Heijden, "User acceptance of hedonic information systems", MIS Quarterly, 28(4), 2004, pp. 695704.

[17] E. Katz, and J.G. Blumler, The uses of mass communications: Current perspectives on gratifications research, Sage Publishing, 1974. 
[18] C. Longoni, and L. Cian, "Artificial intelligence in utilitarian vs. hedonic contexts: The "word-of-machine" effect", Journal of Marketing, forthcoming, 2020.

[19] T. Euler, "The current state of creative AI", https://attentionecono.me/creative-artificial-intelligencemusic-writing-video-movies-2017-7e9c192594a3, accessed 03.03.2021.

[20] A. Graefe, "Guide to automated journalism", https://academiccommons.columbia.edu/doi/10.7916/D8QZ2 P7C/download, accessed 05.11.2020.

[21] E. Jussupow, I. Benbasat, and A. Heinzl, "Why are we averse towards algorithms? A comprehensive literature review on algorithm aversion", Proceedings of the 28th European Conference on Information Systems, 2020.

[22] A.S. Jago, "Algorithms and authenticity", Academy of Management Discoveries, 5(1), 2019, pp. 38-56.

[23] M. Yeomans, A. Shah, S. Mullainathan, and J. Kleinberg, "Making sense of recommendations", Journal of Behavioral Decision Making, 32(4), 2019, pp. 403-414.

[24] Y.E. Bigman, and K. Gray, "People are averse to machines making moral decisions", Cognition, 181, 2018, pp. 21-34.

[25] M. Carlson, "Automating judgment? Algorithmic judgment, news knowledge, and journalistic professionalism", New Media \& Society, 20(5), 2018, pp. 1755-1772.

[26] A. Williams, I. Sherman, S. Smarr, B. Posadas, and J.E. Gilbert, "Human trust factors in image analysis", Proceedings of the International Conference on Applied Human Factors and Ergonomics, 2019.

[27] L. Lohoff, and A. Rühr, "Introducing (machine) learning ability as antecedent of trust in intelligent systems", Proceedings of the 29th European Conference on Information Systems, 2021.

[28] N. Thurman, J. Moeller, N. Helberger, and D. Trilling, "My friends, editors, algorithms, and I: Examining audience attitudes to news selection", Digital Journalism, 7(4), 2019, pp. 447-469.

[29] D.L. Goodhue, and R.L. Thompson, "Task-technology fit and individual performance", MIS Quarterly, 19(2), 1995, pp. 213-236.

[30] B. Liu, and L. Wei, "Machine authorship in situ", Digital Journalism, 7(5), 2019, pp. 635-657.

[31] T.F. Waddell, "Can an algorithm reduce the perceived bias of news?", Journalism \& Mass Communication Quarterly, 96(1), 2019, pp. 82-100.

[32] J. Jung, H. Song, Y. Kim, H. Im, and S. Oh, "Intrusion of software robots into journalism: The public's and journalists' perceptions of news written by algorithms and human journalists", Computers in Human Behavior, 71, 2017, pp. 291-298.

[33] A. Graefe, M. Haim, B. Haarmann, and H.-B. Brosius, "Readers' perception of computer-generated news: Credibility, expertise, and readability", Journalism, 19(5), 2018, pp. 595-610.

[34] A.M. Lee, "News audiences revisited: Theorizing the link between audience motivations and news consumption", Journal of Broadcasting \& Electronic Media, 57(3), 2013, pp. 300-317.

[35] R. Kivetz, O. Urminsky, and Y. Zheng, "The goalgradient hypothesis resurrected: Purchase acceleration, illusionary goal progress, and customer retention", Journal of Marketing Research, 43(1), 2006, pp. 39-58.

[36] S. Arora, F. Hofstede, and V. Mahajan, "The implications of offering free versions for the performance of paid mobile apps", Journal of Marketing, 81(6), 2017, pp. 62-78.

[37] A. Miroshnichenko, "AI to bypass creativity. Will robots replace journalists? (The answer is "yes")", Information, 9(7), 2018, pp. 183-203.

[38] L.N. Lemelshtrich, Robot journalism: Can human journalism survive?, World Scientific, 2018.

[39] X. Luo, S. Tong, Z. Fang, and Z. Qu, "Frontiers: Machines vs. humans: The impact of artificial intelligence chatbot disclosure on customer purchases", Marketing Science, 38(6), 2019, pp. 937-947.

[40] A. Wölker, and T.E. Powell, "Algorithms in the newsroom? News readers' perceived credibility and selection of automated journalism", Journalism, 22(1), 2018.

[41] R. Pennington, H.D. Wilcox, and V. Grover, "The role of system trust in business-to-consumer transactions", Journal of Management Information Systems, 20(3), 2003, pp. 197-226. [42] L. Xu, J. Lin, and H.C. Chan, "The moderating effects of utilitarian and hedonic values on information technology continuance", ACM Transactions on Computer-Human Interaction (TOCHI), 19(2), 2012, pp. 1-26.

[43] T. Holtgraves, S.J. Ross, C. Weywadt, and T. Han, "Perceiving artificial social agents", Computers in Human Behavior, 23(5), 2007, pp. 2163-2174.

[44] R.M. Schuetzler, M. Grimes, J.S. Giboney, and J. Buckman, "Facilitating natural conversational agent interactions: Lessons from a deception experiment", Proceedings of the 35th International Conference on Information Systems, 2014.

[45] R. Agarwal, and J. Prasad, "A conceptual and operational definition of personal innovativeness in the domain of information technology", Information Systems Research, 9(2), 1998, pp. 204-215.

[46] G.L. Polites, and E. Karahanna, "Shackled to the status quo: The inhibiting effects of incumbent system habit, switching costs, and inertia on new system acceptance", MIS Quarterly, 2012, pp. 21-42.

[47] J.F. Hair, C.M. Ringle, and M. Sarstedt, "PLS-SEM: Indeed a silver bullet", Journal of Marketing Theory and Practice, 19(2), 2011, pp. 139-152.

[48] A. Field, Discovering statistics using IBM SPSS statistics, Sage Publishing, 2013.

[49] A. Bhattacherjee, "Understanding information systems continuance: An expectation-confirmation model", MIS Quarterly, 25(3), 2001, pp. 351-370.

[50] W. Wang, and I. Benbasat, "Recommendation agents for electronic commerce: Effects of explanation facilities on trusting beliefs", Journal of Management Information Systems, 23(4), 2007, pp. 217-246.

[51] S. Garfinkel, J. Matthews, S.S. Shapiro, and J.M. Smith, "Toward algorithmic transparency and accountability", Communications of the ACM, 60(9), 2017.

[52] P. Nussbaumer, I. Matter, and G. Schwabe, "“Enforced" vs. "casual" transparency - Findings from IT-supported financial advisory encounters", ACM Transactions on Management Information Systems, 3(2), 2012, pp. 1-19. 